\title{
Predicting outcomes in traumatic out-of-hospital cardiac arrest: the relevance of Utstein factors
}

\author{
Ben Beck, ${ }^{1}$ Janet E Bray, ${ }^{1,2,3}$ Peter Cameron, ${ }^{1,3}$ Lahn Straney, ${ }^{1}$ Emily Andrew, ${ }^{1,4}$ \\ Stephen Bernard, ${ }^{1,4,5}$ Karen Smith ${ }^{1,4,6}$
}

\begin{abstract}
Additional material is published online only. To view, please visit the journal online (http://dx.doi.org/10.1136/ emermed-2016-206330).
\end{abstract}

1 Department of Epidemiology and Preventive Medicine, School of Public Health \& Preventive Medicine, Monash University, Melbourne, Victoria, Australia ${ }^{2}$ Prehospital, Resuscitation and Emergency Care Research Unit (PRECRU), School of Nursing, Midwifery and Paramedicine,

Curtin University, Perth, Western Australia, Australia

${ }^{3}$ Emergency and Trauma Centre, The Alfred Hospital, Melbourne, Victoria, Australia

${ }^{4}$ Centre for Research and Evaluation, Ambulance Victoria, Melbourne, Victoria, Australia ${ }^{5}$ Intensive Care Unit, The Alfred Hospital, Melbourne, Victoria Australia

${ }^{6}$ Department of Community Emergency Health and Paramedic Practice, MonashUniversity, Melbourne, Victoria, Australia

\section{Correspondence to} Dr Ben Beck, Department of Epidemiology and Preventive Medicine, Monash University, The Alfred Centre, 99 Commercial Rd, Melbourne, VIC 3004, Australia;

ben.beck@monash.edu

Received 5 September 2016 Revised 30 January 2017

Accepted 19 July 2017 Published Online First 11 August 2017

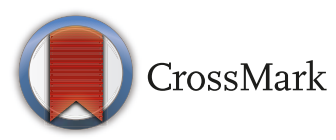

To cite: Beck $B$, Bray JE, Cameron $\mathrm{P}$, et al. Emerg Med $J$ 2017:34:786-792.

\section{ABSTRACT \\ Background Given low survival rates in cases of} traumatic out-of-hospital cardiac arrest (OHCA), there is a need to identify factors associated with outcomes. We aimed to investigate Utstein factors associated with achieving return of spontaneous circulation (ROSC) and survival to hospital in traumatic OHCA.

Methods The Victorian Ambulance Cardiac Arrest Registry (VACAR) was used to identify cases of traumatic OHCA that received attempted resuscitation and occurred between July 2008 and June 2014. We excluded cases aged $<16$ years or with a mechanism of hanging or drowning.

Results of the 660 traumatic OHCA patients who received attempted resuscitation, ROSC was achieved in 159 patients (24\%) and 95 patients (14\%) survived to hospital (ROSC on hospital handover). Factors that were positively associated with achieving ROSC in multivariable logistic regression models were age $\geq 65$ years (adjusted $O R(A O R)=1.56,95 \% \mathrm{Cl}: 1.01$ to 2.43) and arresting rhythm (shockable $(A O R=3.65$, $95 \% \mathrm{Cl}: 1.64$ to 8.11$)$ and pulseless electrical activity ( $A O R=2.15,95 \% \mathrm{Cl}: 1.36$ to 3.39 ) relative to asystole) Similarly, factors positively associated with survival to hospital were arresting rhythm (shockable $(A O R=3.92$, $95 \% \mathrm{Cl}: 1.64$ to 9.41 ) relative to asystole), and the mechanism of injury (falls ( $A O R=2.16,95 \% \mathrm{Cl}: 1.03$ to 4.54 ) relative to motor vehicle collisions), while trauma type (penetrating ( $\mathrm{AOR}=0.27,95 \% \mathrm{Cl}$ : 0.08 to 0.91 ) relative to blunt trauma) and event region (rural ( $A O R=0.39,95 \% \mathrm{Cl}: 0.19$ to 0.80 ) relative to urban) were negatively associated with survival to hospital. Conclusions Few patient and arrest characteristics were associated with outcomes in traumatic OHCA. These findings suggest there is a need to incorporate additional information into cardiac arrest registries to assist prognostication and the development of novel interventions in these trauma patients.

\section{INTRODUCTION}

Survival from cases of traumatic out-of-hospital cardiac arrest (OHCA) is generally reported to be low, with a recent systematic review reporting an overall survival of $3.3 \% .^{1}$ Despite this, there has been an emerging interest in traumatic OHCA. However, there remains little known about the characteristics that are associated with patient outcomes. This is in contrast to cases of medical OHCA in which prognostic factors are well established; these include response time, the arrest being witnessed, the presence of bystander cardiopulmonary resuscitation (CPR) and an initial rhythm that is shockable. ${ }^{2}$

\section{Key messages}

What is already known on this subject?

- Cardiac arrest variables are reported using the Utstein style, a set of variables that have been used to determine prognostic factors in cardiac arrest. However, whether those variables are predictive of outcome in traumatic out of hospital arrest is not known.

What this study adds?

- Using data extracted from the Victorian Cardiac Arrest Registry, we found Utsein characteristics predictive of achieving ROSC and survival to hospital. Arresting rhythm was the only variable associated with positive associations for both of these outcomes in multivariate models. Specifically, response time, witnessed arrest, and bystander CPR were not associated with outcomes. There is a need to include additional information in cardiac arrest registries, particularly detailed injury data.

Prior studies in traumatic OHCA have reported descriptive statistics of those who achieved return of spontaneous circulation (ROSC) or those who survived to hospital discharge. ${ }^{3-7}$ In the absence of multivariable models, it is difficult to determine which factors are associated with patient survival and which may be confounded by other factors. Only two prior studies have used multivariable models in traumatic $\mathrm{OHCA}^{3}{ }^{6}$; however, these studies focused on the impact of advanced interventions, such as airway device type and chest tube insertion, or physiological measures. As a result, there is limited evidence available to ascertain whether known prognostic factors in medical aetiology, including key Utstein variables, ${ }^{8}$ are also predictive in traumatic aetiology.

In this study, we aim to investigate factors that are associated with outcomes from traumatic OHCA using multivariable logistic regression models. Specifically, we aim to focus on Utstein factors associated with achieving prehospital ROSC and having ROSC on arrival at hospital.

\section{METHODS}

\section{Study design}

Retrospective data were extracted from the Victorian Ambulance Cardiac Arrest Registry (VACAR). We included those who received attempted resuscitation by paramedics, were aged $>15$ years, 
experienced an OHCA of traumatic aetiology (excluding those with a mechanism of hanging or drowning) and suffered an arrest between 1 July 2008 and 30 June 2014.

\section{Setting}

The study was conducted in the state of Victoria, Australia, which has a population of approximately 5.6 million people, ${ }^{9} 75 \%$ of whom reside in the metropolitan region of Melbourne. Ambulance Victoria (AV) is the sole provider of emergency medical services (EMS) in the state. AV delivers a two-tiered EMS system, with Advanced Life Support Paramedics (ALS) and Intensive Care Ambulance Paramedics (ICP). While physicians are used for secondary response (retrieval), they do not attend as part of the primary response. ALS paramedics are capable of laryngeal mask airway insertion, manual defibrillation, decompression of suspected tension pneumothorax in rural areas and intravenous fluid and epinephrine administration. ICP paramedics, in addition to ALS skills, are authorised to perform endotracheal intubation, rapid sequence induction, Pneumocath insertion and administer a wider range of drugs. The administration of prehospital blood products was introduced in April 2011 for the ICP paramedic-staffed helicopter emergency medical service. Prehospital thoracotomies are not performed. Fire fighters and volunteer Community Emergency Response Teams provide a first response in select areas of Victoria. Paramedic treatment guidelines follow the recommendations of the Australian Resuscitation Council (www.resus.org.au). During the study period, the treatment of traumatic OHCA followed ALS guidelines for presumed cardiac OHCA, with the addition of standard trauma management. Criteria for withholding or ceasing resuscitation attempts have previously been described.$^{10}$ In short, paramedics can withhold resuscitation when there is clear evidence of prolonged cardiac arrest or when injuries are incompatible with life.

\section{Victorian Ambulance Cardiac Arrest Registry}

The VACAR is a population-based registry of all OHCA events attended by EMS in the state of Victoria, Australia. The registry captures in-field treatment data electronically and a highly sensitive search filter is used to identify potential cardiac arrest cases before manual review by registry personnel. The registry methodology, including data capture and completeness, and quality assurance processes have been described previously. ${ }^{11}$ The Victorian Department of Health Human Research Ethics Committee (HREC) has approved VACAR data collection (No. 08/02). Ethics approval for the current study was received from the Monash University Human Research Ethics Committee (CF15/3030-2015001273).

\section{Definitions}

ROSC was defined as any palpable pulse detected for $>20$ s during treatment by paramedics in the prehospital setting. Survival to hospital was defined as the patient having ROSC on handover from paramedics at hospital. VACAR defines 'prolonged downtime' as cases where the duration between collapse and arrival of EMS is presumed to be $>15 \mathrm{~min}$. The Australian Bureau of Statistics' Section of State (SOS) classification ${ }^{12}$ was used to define whether the arrest occurred in an urban or rural location. As per the SOS classification, urban centre and locality (UCL) regions with a population of $<1000$ persons were classified as rural. Age was dichotomised into those $<65$ years and $\geq 65$ years. Trauma type was classified as blunt or penetrating. For the multivariable models, injury mechanism was collapsed into seven categories: motor vehicle collision, motorcycle collision, pedestrian, fall, shooting/stabbing, other or unknown. Due to low numbers in the subcategories of falls (low falls: $\mathrm{n}=48$, high falls: $\mathrm{n}=24$, stairs: $\mathrm{n}=5$, other: $n=6$ ), these were included as one group. The other category in the multivariable models included assaults $(20 \%)$, crush events (20\%), bicycle collisions (15\%), train collision (pedestrian) (11\%), fire exposure (9\%) and industrial events $(7 \%)$.

\section{Statistical analysis}

Comparisons between cases that achieved ROSC and those that did not achieve ROSC were made using the $\chi^{2}$ test and the MannWhitney $\mathrm{U}$ test, as appropriate. Univariate and multivariable logistic regression models were used to investigate patient and arrest factors associated with two outcomes: achieving ROSC and survival to hospital (ROSC on hospital handover). The Hosmer-Lemeshow $\chi^{2}$ test was used to assess goodness of fit of the model. A sensitivity analysis was conducted for both models to determine whether the exclusion of non-standard Utstein variables $^{8}$ (region, prolonged downtime, trauma type and mechanism of injury) impacted on the significance of core Utstein variables. Data analysis was performed using Stata (V.13.1, StataCorp, College Station, Texas, USA). A P value $<0.05$ was considered to be statistically significant.

\section{RESULTS}

\section{Baseline characteristics}

From July 2008 to June 2014, paramedics attended 2334 cases of traumatic OHCA, of which $660(28 \%)$ received attempted resuscitation. ICP paramedics were present in $584(88 \%)$ of those cases that received attempted resuscitation. Patients who received attempted resuscitation had a median age of 42.5 years (IQR: 28-61), were predominantly male $(72 \%)$, with their arrests most commonly occurring in a public place $(71 \%)$ and resulting from blunt trauma (81\%). Motor vehicle collisions (28\%), motorcycle collisions (18\%), pedestrian collisions (14\%) and falls (13\%) were the most common mechanism of injury that resulted in traumatic OHCA (table 1).

ROSC was achieved in 159 patients, representing 24\% of the EMS-treated population. Comparisons between those achieving and not achieving ROSC (table 1) revealed that ROSC was achieved more commonly among arrests witnessed by EMS, and less commonly among cases of asystolic arrest.

\section{Factors associated with achieving ROSC}

Events with age $\geq 65$ years, witnessed status (an arrest witnessed by EMS relative to unwitnessed arrests), the arrest rhythm (shockable rhythms or pulseless electrical activity (PEA) relative to asystole), and the mechanism of injury (falls relative to motor vehicle collisions) were associated with an increased likelihood of achieving ROSC in univariate models (table 2). Events occurring in rural locations, the presence of bystander CPR and prolonged downtime were negatively associated with achieving ROSC in univariate models. When cases were limited to only those that were bystander-witnessed, the presence of bystander CPR was not associated with achieving ROSC in a univariate model $(\mathrm{OR}=0.69,95 \% \mathrm{CI}: 0.40$ to $1.19, \mathrm{p}=0.19)$. Multivariable models revealed that older age and arresting rhythm (shockable rhythms and PEA relative to asystole) were positively associated with achieving ROSC (table 2). 
Table 1 Baseline characteristics of the study sample. Data are presented as overall, and then stratified by cases in which ROSC was and was not achieved. Percentages for categorical variables represent proportions within groupings for the column describing all attempted resuscitation cases, while percentages comparing cases where ROSC was achieved reflect the individual row

\begin{tabular}{|c|c|c|c|c|c|}
\hline \multirow[b]{2}{*}{ Grouping } & \multirow[b]{2}{*}{ Characteristic } & \multirow[b]{2}{*}{ All attempted resuscitation } & \multicolumn{2}{|l|}{ ROSC achieved } & \multirow[b]{2}{*}{$P$ value } \\
\hline & & & ROSC achieved & No ROSC achieved & \\
\hline & Number of cases & 660 & $159(24.1 \%)$ & $501(75.9 \%)$ & \\
\hline & Age, median (IQR) & $42.5(28-61)$ & $53(29-72)$ & $40(27-59)$ & $<0.001$ \\
\hline \multirow[t]{4}{*}{ Sex, n (\%) } & & & & & 0.666 \\
\hline & Male & $476(72.1 \%)$ & $111(23.3 \%)$ & $365(76.7 \%)$ & \\
\hline & Female & $157(23.8 \%)$ & $42(26.8 \%)$ & $115(73.2 \%)$ & \\
\hline & Indeterminate/unknown & $27(4.1 \%)$ & $6(22.2 \%)$ & $21(77.8 \%)$ & \\
\hline \multirow[t]{4}{*}{ Location, n (\%) } & & & & & $<0.001$ \\
\hline & Public place & $466(70.6 \%)$ & $93(20.0 \%)$ & $373(80.0 \%)$ & \\
\hline & Private residence & $112(17.0 \%)$ & $34(30.4 \%)$ & $78(69.6 \%)$ & \\
\hline & Other/unknown & $82(12.4 \%)$ & $32(39.0 \%)$ & $50(60.1 \%)$ & \\
\hline \multirow[t]{4}{*}{ Region, n (\%) } & & & & & 0.036 \\
\hline & Urban & $464(70.3 \%)$ & $124(26.7 \%)$ & $340(73.3 \%)$ & \\
\hline & Rural & $192(29.1 \%)$ & $35(18.2 \%)$ & $157(81.8 \%)$ & \\
\hline & Unknown & $4(0.6 \%)$ & $0(0 \%)$ & $4(100 \%)$ & \\
\hline \multirow[t]{4}{*}{ Type of trauma, n (\%) } & & & & & 0.375 \\
\hline & Blunt & $535(81.1 \%)$ & $133(24.9 \%)$ & $402(75.1 \%)$ & \\
\hline & Penetrating & $120(18.2 \%)$ & $24(20.0 \%)$ & $96(80.0 \%)$ & \\
\hline & Unknown & $5(0.8 \%)$ & $2(40.0 \%)$ & $3(60.0 \%)$ & \\
\hline \multirow[t]{12}{*}{ Injury mechanism, n (\%) } & & & & & 0.068 \\
\hline & Motor vehicle collision & $187(28.3 \%)$ & $44(23.5 \%)$ & $143(76.5 \%)$ & \\
\hline & Motorcycle collision & $120(18.2 \%)$ & $18(15.0 \%)$ & $102(85.0 \%)$ & \\
\hline & Pedestrian collision & $90(13.6 \%)$ & $21(23.3 \%)$ & $69(76.7 \%)$ & \\
\hline & Bicycle collision & $15(2.3 \%)$ & $3(20.0 \%)$ & $12(80.0 \%)$ & \\
\hline & Train collision (pedestrian) & $11(1.7 \%)$ & $2(18.2 \%)$ & $9(81.8 \%)$ & \\
\hline & Fall & $83(12.6 \%)$ & $30(36.1 \%)$ & $53(63.9 \%)$ & \\
\hline & Shooting & $29(4.4 \%)$ & $6(20.7 \%)$ & $23(79.3 \%)$ & \\
\hline & Stabbing & $48(7.3 \%)$ & $10(20.8 \%)$ & $38(79.2 \%)$ & \\
\hline & Blunt assault & $20(3.0 \%)$ & $8(40.0 \%)$ & $12(60.0 \%)$ & \\
\hline & Other* & $54(8.2 \%)$ & $16(29.6 \%)$ & $38(70.4 \%)$ & \\
\hline & Unknown & $3(0.5 \%)$ & $1(33.3 \%)$ & $2(66.7 \%)$ & \\
\hline \multirow[t]{5}{*}{ Arrest witnessed by, n (\%) } & & & & & $<0.001$ \\
\hline & Bystander & $329(49.9 \%)$ & $65(19.8 \%)$ & $264(80.2 \%)$ & \\
\hline & EMS & $208(31.5 \%)$ & $72(34.6 \%)$ & $136(65.4 \%)$ & \\
\hline & Unwitnessed & $105(15.9 \%)$ & $20(19.0 \%)$ & $85(81.0 \%)$ & \\
\hline & Unknown & $18(2.7 \%)$ & $2(11.1 \%)$ & $16(88.9 \%)$ & \\
\hline \multicolumn{2}{|c|}{$\begin{array}{l}\text { Bystander CPR initiated in cases where arrest was } \\
\text { witnessed by bystander, } \mathrm{n}(\%)\end{array}$} & $166(50.5 \%)$ & $28(16.9 \%)$ & $138(83.1 \%)$ & 0.184 \\
\hline \multicolumn{2}{|c|}{$\begin{array}{l}\text { Response time for non-EMS witnessed cases, median } \\
\text { (IQR) }\end{array}$} & $9.6(7.5-14.0)$ & $8.5(7.1-11.5)$ & $10(7.5-15.0)$ & 0.011 \\
\hline \multicolumn{2}{|l|}{ Prolonged downtime, n (\%) } & $144(21.8 \%)$ & $17(11.8 \%)$ & $127(88.2 \%)$ & $<0.001$ \\
\hline \multicolumn{2}{|l|}{ Initial arresting rhythm, n (\%) } & & & & $<0.001$ \\
\hline & VF/VT & $37(5.6 \%)$ & $15(40.5 \%)$ & $22(59.5 \%)$ & \\
\hline & Pulseless electrical activity & $334(50.6 \%)$ & $100(29.9 \%)$ & $234(71.1 \%)$ & \\
\hline & Asystole & $274(41.5 \%)$ & $37(13.5 \%)$ & $237(86.5 \%)$ & \\
\hline & Unknown & $15(2.3 \%)$ & $7(29.9 \%)$ & $8(70.1 \%)$ & \\
\hline
\end{tabular}

*The 'other' grouping includes crush events, fire exposure and industrial events.

CPR, cardiopulmonary resuscitation; EMS, emergency medical services; ROSC, return of spontaneous circulation; VF, ventricular fibrillation; VT, ventricular tachycardia.

\section{Factors associated with survival to hospital}

Of those that received an attempted resuscitation, 95 patients (14.4\%) survived to hospital (ROSC on hospital handover). Older age, arrest rhythm (shockable rhythms or PEA relative to asystole) and the mechanism of injury (falls relative to motor vehicle collisions) were associated with an increased likelihood of the patient surviving to hospital in univariate models (table 3 ).
Events occurring in rural locations, increased response time and prolonged downtime were negatively associated with survival to hospital in univariate models. Results of the multivariable model demonstrated arrests occurring in urban locations, the arresting rhythm (shockable rhythms compared with asystole), and the mechanism of injury (falls relative to motor vehicle collisions) were associated with an increased odds of survival to hospital 
Table 2 Logistic regression model with the presence of prehospital ROSC as the binary outcome $(\mathrm{n}=636)$. Results are presented as univariate and multivariable analyses

\begin{tabular}{|c|c|c|c|c|}
\hline & Univariate OR $(95 \% \mathrm{Cl})$ & $P$ value & Adjusted OR (95\% Cl) & $P$ value \\
\hline Age $\geq 65$ years & 1.87 (1.27 to 2.74$)$ & 0.002 & 1.56 (1.01 to 2.43$)$ & 0.047 \\
\hline \multicolumn{5}{|l|}{ Region } \\
\hline Urban & Reference & & Reference & \\
\hline Rural & 0.61 (0.40 to 0.93 ) & 0.022 & 0.83 (0.50 to 1.39$)$ & 0.483 \\
\hline \multicolumn{5}{|l|}{ Witnessed } \\
\hline Unwitnessed/unknown & Reference & & Reference & \\
\hline Bystander & 1.13 (0.66 to 1.93$)$ & 0.654 & 1.04 (0.55 to 1.95$)$ & 0.904 \\
\hline EMS & 2.43 (1.41 to 4.18$)$ & 0.001 & 1.55 (0.76 to 3.15$)$ & 0.227 \\
\hline Bystander CPR* & 0.49 (0.32 to 0.74$)$ & 0.001 & 0.71 (0.42 to 1.19$)$ & 0.189 \\
\hline \multicolumn{5}{|l|}{ Rhythm } \\
\hline Asystole & Reference & & Reference & \\
\hline VF/VT & 4.37 (2.08 to 9.17 ) & $<0.001$ & 3.65 (1.64 to 8.11$)$ & 0.001 \\
\hline PEA & 2.74 (1.80 to 4.16$)$ & $<0.001$ & 2.15 (1.36 to 3.39 ) & 0.001 \\
\hline \multicolumn{5}{|l|}{ Response timet } \\
\hline $0-10 \mathrm{~min}$ & Reference & & Reference & \\
\hline $11-20 \mathrm{~min}$ & 0.46 (0.29 to 0.73$)$ & 0.001 & 0.83 (0.45 to 1.50$)$ & 0.529 \\
\hline$>20$ min & 0.28 (0.11 to 0.73$)$ & 0.009 & 0.70 (0.22 to 2.26$)$ & 0.552 \\
\hline Prolonged downtime & 0.35 (0.21 to 0.61$)$ & $<0.001$ & $0.59(0.29$ to 1.20$)$ & 0.147 \\
\hline \multicolumn{5}{|l|}{ Trauma type } \\
\hline Blunt & Reference & & Reference & \\
\hline Penetrating & 0.76 (0.46 to 1.23$)$ & 0.261 & 0.57 (0.24 to 1.31$)$ & 0.183 \\
\hline \multicolumn{5}{|l|}{ Mechanism of injury } \\
\hline Motor vehicle collision & Reference & & Reference & \\
\hline Motorcycle collision & 0.57 (0.31 to 1.05$)$ & 0.071 & 0.72 (0.37 to 1.38 ) & 0.321 \\
\hline Pedestrian & 0.99 (0.55 to 1.79$)$ & 0.971 & 0.97 (0.50 to 1.86$)$ & 0.920 \\
\hline Fall & 1.84 (1.05 to 3.22$)$ & 0.033 & 1.78 (0.93 to 3.42$)$ & 0.081 \\
\hline Shooting/stabbing & 0.85 (0.45 to 1.63$)$ & 0.628 & 1.64 (0.57 to 4.68$)$ & 0.358 \\
\hline Other & 1.33 (0.77 to 2.30$)$ & 0.311 & 1.86 (1.00 to 3.46$)$ & 0.050 \\
\hline Unknown & $1.63(0.14$ to 18.35$)$ & 0.695 & $6.68(0.32$ to 137.3$)$ & 0.219 \\
\hline
\end{tabular}

* Bystander CPR was coded as 0 when the arrest was witnessed by EMS.

† Response time was coded as 0 when the arrest was witnessed by EMS.

CPR, cardiopulmonary resuscitation; EMS, emergency medical services; ROSC, return of spontaneous circulation; PEA, pulseless electrical activity; VF, ventricular fibrillation; VT, ventricular tachycardia.

(table 3). Trauma type (penetrating trauma compared with blunt trauma) was associated with a decreased odds of survival to hospital. Goodness-of-fit tests were not statistically significant for the models of achieving ROSC (Hosmer-Lemeshow $\chi^{2}=9.26, \mathrm{P}=0.32$ ) or survival to hospital (Hosmer-Lemeshow $\left.\chi^{2}=9.57, \mathrm{P}=0.30\right)$ indicating adequate model fit across deciles of risk.

\section{Sensitivity analysis}

The sensitivity analysis revealed that, for most variables, there was no change in the significance or direction of association between outcomes and the Utstein variables (witnessed status, the presence of bystander CPR, the arresting rhythm and response time) when excluding non-standard Utstein variables (region, prolonged downtime, trauma type and mechanism of injury) (see online supplementary appendix 1). The exception was age, which was non-significant in the multivariable model for survival to hospital when non-standard Utstein variables were included, however, became significant when the multivariable model was limited to the Utstein variables.

\section{Survival to hospital discharge}

Of those patients who achieved prehospital ROSC, 15.1\% $(n=24)$ survived to hospital discharge. A single patient who did not achieve prehospital ROSC survived to hospital discharge. The 25 patients who survived to hospital discharge represented $3.8 \%$ of those who received attempted resuscitation and $1.1 \%$ of the total traumatic OHCA population.

\section{DISCUSSION}

The purpose of this study was to investigate factors associated with achieving ROSC and survival to hospital in a cohort of traumatic OHCA patients. Our results demonstrated limited predictors of these outcomes; arresting rhythm was the only variable that demonstrated positive associations with outcomes across both multivariable models. These results suggest that known prognostic factors in medical aetiology may not apply in traumatic aetiology. In order to inform prognostication, there is a need to identify other factors that may be associated with outcomes in traumatic OHCA.

This is the first study to use multivariable models to identify patient and arrest characteristics that are associated with outcomes from traumatic OHCA. Previous studies have used descriptive statistics to characterise a cohort of traumatic OHCA patients that survived to hospital ${ }^{4}$ or hospital discharge, ${ }^{13}$ or compared survivors and non-survivors. ${ }^{3}$ Pickens et $a l^{5}$ noted no differences in demographics and the mechanism of injury between survivors and non-survivors. This was supported by 
Table 3 Logistic regression model with survival to hospital (ROSC on hospital handover) as the outcome ( $\mathrm{n}=636$ ). Results are presented as univariate and multivariable analyses

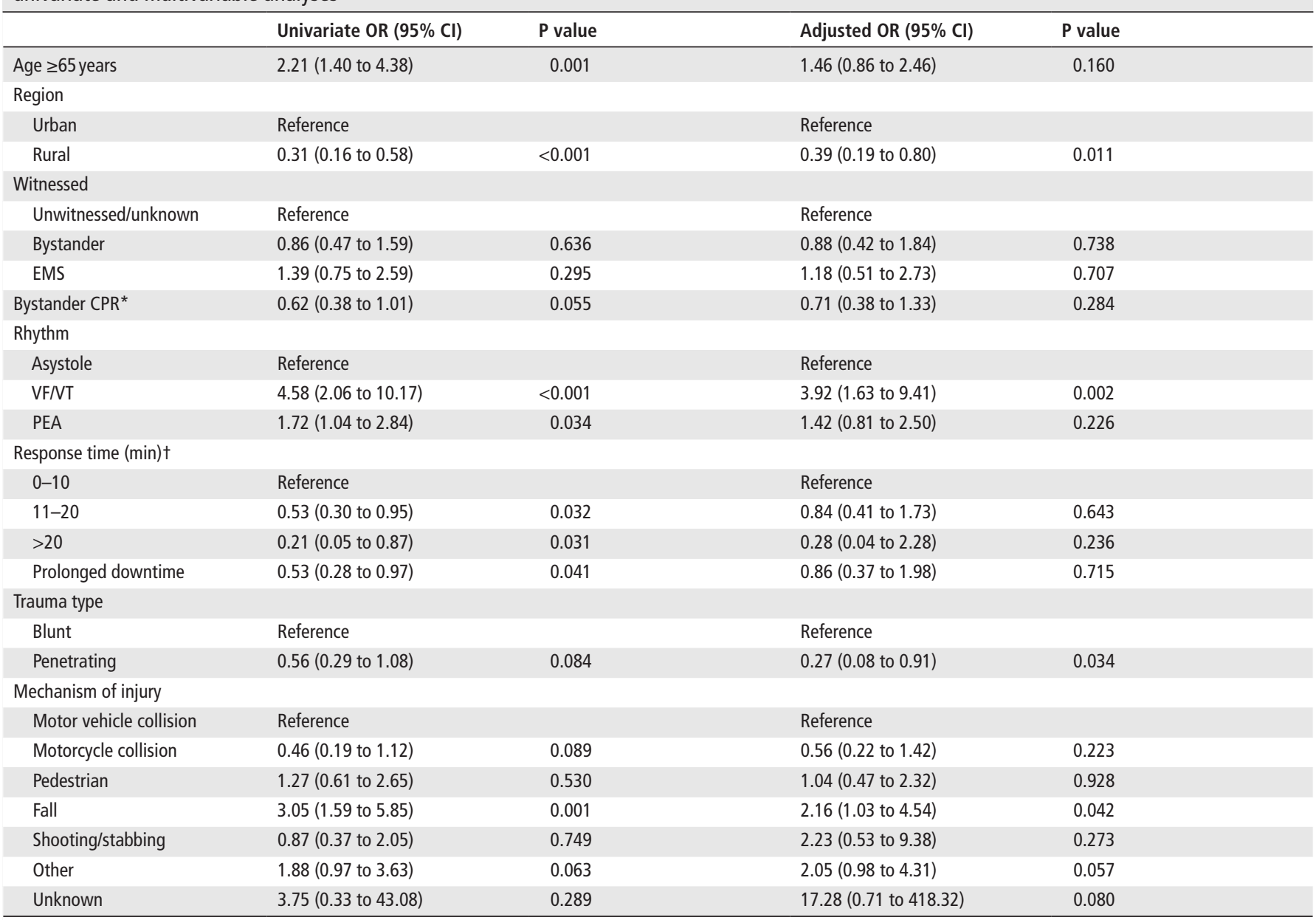

* Bystander CPR was coded as 0 when the arrest was witnessed by EMS.

tResponse time was coded as 0 when the arrest was witnessed by EMS.

CPR, cardiopulmonary resuscitation; EMS, emergency medical services; ROSC, return of spontaneous circulation; PEA, pulseless electrical activity; VF, ventricular fibrillation; VT, ventricular tachycardia.

Evans et al $^{3}$ who demonstrated few differences between survivors and non-survivors using data from the Resuscitation Outcomes Consortium Epistry-Trauma and PROPHET registries; only the mechanism of injury and injury type differed between groups. Our findings that there were few predictors of outcomes in traumatic OHCA are consistent with these prior studies.

The major strength of our study is the use of multivariable models that enabled us to control for potential sources of confounding. These models demonstrated that older age was positively associated with achieving ROSC; a finding that has not previously been reported and is in contrast to cases of medical OHCA. ${ }^{11}$ Two prior studies in traumatic OHCA demonstrated no difference in age between survivors and non-survivors, ${ }^{35}$ while another demonstrated greater mortality in older patients. ${ }^{6}$ When controlling for injury severity, mortality is known to increase with increasing age in trauma patients ${ }^{14}$ and this has been suggested to result from pre-existing conditions or complications. ${ }^{15}$ In our study, we focused on early outcomes, rather than long-term outcomes such as survival to hospital discharge. Our finding that older patients were more likely to achieve ROSC may suggest that these patients have a lower threshold for cardiac arrest. Furthermore, a proportion of these patients may have been in a low-flow state in which a pulse was not palpable, but mechanical and electrical cardiac activity were still present. Further research is required to understand the age-related response and whether it relates to the mechanism, a concomitant medical event or the severity of injuries sustained. Consistent with the age-related association with achieving ROSC was our finding that patients with an OHCA following a fall had an increased odds of surviving to hospital when compared with those following a motor vehicle collision. It is known that patients with fall-related trauma are typically older, have a lower injury severity score (ISS) when compared with motor vehicle collisions, ${ }^{16}$ and are the most likely traumatic OHCA patients to survive to hospital discharge. ${ }^{3}$ This supports our hypothesis that specific patient subgroups may have a lower threshold for traumatic cardiac arrests. It is possible that a proportion of these falls may have been induced by a cardiovascular event and are not primary traumatic arrests. This may also be true for a proportion of motor vehicle collisions, particularly the cases with a shockable rhythm. However, in the absence of detailed injury information, we could not determine the pathophysiological cause of their arrest or whether the older trauma patients had a lower injury severity score.

Conjecture currently exists as to whether blunt or penetrating trauma results in poorer outcomes. While some studies have showed no differences in outcomes between trauma types, ${ }^{5} 17$ our results demonstrated that blunt trauma was associated with 
an increased odds of survival to hospital relative to penetrating trauma. This finding is consistent with recent North American data. ${ }^{3}$ However, the reasons for this are not clear. Understanding the specific injuries in the blunt and penetrating trauma groups may facilitate greater understanding of this finding; however, this was not possible in the current study.

In contrast to our results that demonstrated limited factors associated with outcomes in traumatic OHCA, the predictors of outcome in medical OHCA are well established ${ }^{2}$ and have informed the development of the 'chain of survival'. ${ }^{18}$ These predictors include the presence of bystander CPR and the arrest being witnessed by a bystander or EMS, both of which we showed to have no association with outcomes from traumatic OHCA. While bystander CPR rates in our region were higher than have been previously reported for traumatic OHCA, ${ }^{4}$ the utility of bystander CPR in traumatic cardiac arrest has been questioned $^{719}$ and our results demonstrated that the presence of bystander CPR was not associated with achieving ROSC or survival to hospital in multivariable models. The presence of bystander CPR may also increase the likelihood of a paramedic attempting resuscitation in cases that are potentially futile. However, it was not possible to quantify this in our study.

In order to capture key patient and arrest elements in medical OHCA, the Utstein template was developed. ${ }^{8}$ This was originally developed in 1990 with a focus on non-EMS witnessed cardiac arrests of a presumed cardiac cause with a rhythm of ventricular fibrillation. This was expanded in 2004 to include all EMS-treated cardiac arrests. While these factors facilitate a clear understanding of the impact of patient and arrest characteristics on outcomes in medical OHCA, our results suggest that the Utstein template may not provide sufficient information to predict outcomes in traumatic OHCA. While our models also included variables outside of the current Ustein template, such as urban/rural location, injury type and injury mechanism, our sensitivity analyses that excluded these non-standard Utstein variables revealed that, with the exception of age in our model of survival to hospital, there was no change in the significance or direction of association between standard Utstein variables and outcomes from traumatic OHCA. Given the results of our study, there is a need to incorporate other variables that may facilitate a greater understanding of outcomes and assist in prognosticating in traumatic OHCA. The most obvious addition is that of detailed injury information. This is possible in regions where established trauma registries exist, such as in Germany ${ }^{6}$ and the UK, ${ }^{20}$ and is also possible in our region through linkage with the Victorian State Trauma Registry. However, this trauma registry is limited to those patients who are transported to hospital. Obtaining detailed injury information for those who are pronounced dead at the scene is challenging due to an inability to appropriately quantify and classify injuries. In these cases, obtaining detailed injury data is only possible through coronial data. This has previously been conducted in reviewing potentially preventable prehospital deaths. ${ }^{21}$ However, to our knowledge, combining coronial data for prehospital deaths with in-hospital injury information for those transported to hospital has not been conducted in traumatic OHCA. Regardless, there is a need to identify other data that may facilitate a greater understanding of factors associated with outcomes from traumatic OHCA and subsequently inform the development of novel interventions. Other Ustein-like consensus guidelines have been developed in patient subgroups, such as drowning, ${ }^{22}$ and major trauma ${ }^{23}$; a similar consensus document may be valuable for the subgroup of cardiac arrests resulting from trauma.

\section{Limitations}

There are a number of limitations to consider when reviewing the results of this study. First, we had originally aimed to investigate factors associated with survival from traumatic OHCA. However, with only 25 survivors to hospital discharge, we were underpowered for any multivariable analyses. Low numbers of survivors is a problem that persists throughout the literature on traumatic OHCA, ${ }^{34713}$ and subsequently, we focused on known predictors of survival; namely achieving ROSC and survival to hospital. Second, there was limited prehospital injury information, such as an injury severity score. As this is also a common limitation across the traumatic OHCA literature, it potentially limits our ability to more accurately prognosticate in these patients. It is an area of research that we hope to address in the future through linkage to detailed coronial data. Additionally, specific guidelines for the management of traumatic OHCA have recently been provided by both the Australian Resuscitation Council $^{24}$ and the International Liaison Committee on Resuscitation. ${ }^{25}$ However, these guidelines were released after the end of the study period and as such, were not implemented in clinical practice during our study.

\section{CONCLUSION}

This study of traumatic OHCA patients who received attempted resuscitation demonstrated that there were limited patient and arrest characteristics that were associated with achieving ROSC or survival to hospital. Given that predictors of outcomes in medical OHCA may not apply in traumatic OHCA, there is a need to further develop cardiac arrest registries to incorporate additional information, particularly detailed injury information, which may help guide prognostication and the development of novel interventions in these trauma patients.

\section{Twitter@DrBenBeck}

Contributors $B B, J B, P C, L S, E A, S B, K S$ contributed to the design and planning of the study. $B B$ and $L S$ performed the analysis. $B B, J B, P C, L S, E A, S B, K S$ drafted the manuscript.

Funding $B B, J B$ and $L S$ receive salary support by the National Health and Medical Research Council (NHRMC) Australian Resuscitation Outcomes Consortium (AusROC) Centre of Research Excellence \#1029983 https://www.ausroc.org.au/. JB is supported by a co-funded NHMRC/National Heart Foundation (NHF) Fellowship (\#1069985). Peter Cameron was supported by a NHMRC Practitioner Fellowship (\#545926).

Competing interests None declared.

Ethics approval Monash University Human Research Ethics Committee.

Provenance and peer review Not commissioned; externally peer reviewed.

(c) Article author(s) (or their employer(s) unless otherwise stated in the text of the article) 2017. All rights reserved. No commercial use is permitted unless otherwise expressly granted.

\section{REFERENCES}

1 Zwingmann J, Mehlhorn AT, Hammer T, et al. Survival and neurologic outcome after traumatic out-of-hospital cardiopulmonary arrest in a pediatric and adult population: a systematic review. Crit Care 2012;16:R117.

2 Sasson C, Rogers MA, Dahl J, et al. Predictors of survival from out-of-hospital cardiac arrest: a systematic review and meta-analysis. Circ Cardiovasc Qual Outcomes 2010;3:63-81

3 Evans CC, Petersen A, Meier EN, et al. Prehospital traumatic cardiac arrest: Management and outcomes from the resuscitation outcomes consortium epistrytrauma and PROPHET registries. J Trauma Acute Care Surg 2016;81:285-93.

4 Gräsner JT, Wnent J, Seewald S, et al. Cardiopulmonary resuscitation traumatic cardiac arrest--there are survivors. An analysis of two national emergency registries. Crit Care 2011;15:R276-10.

5 Pickens JJ, Copass MK, Bulger EM. Trauma patients receiving CPR: predictors of survival. J Trauma 2005;58:951-8.

6 Huber-Wagner S, Lefering R, Qvick M, et al. Outcome in 757 severely injured patients with traumatic cardiorespiratory arrest. Resuscitation 2007;75:276-85. 
7 Beck B, Tohira H, Bray JE, et al. Trends in traumatic out-of-hospital cardiac arrest in Perth, Western Australia from 1997 to 2014. Resuscitation 2016;98:79-84.

8 Perkins GD, Jacobs IG, Nadkarni VM, et al. Cardiac arrest and cardiopulmonary resuscitation outcome reports: update of the utstein resuscitation registry templates for out-of-hospital cardiac arrest: A statement for healthcare professionals from a task force of the International liaison committee on resuscitation (American heart association, European resuscitation council, Australian and New Zealand council on resuscitation, heart and stroke foundation of Canada, InterAmerican heart foundation, resuscitation council of Southern Africa, resuscitation council of Asia); and the American heart association emergency cardiovascular care committee and the council on cardiopulmonary, critical care, perioperative and resuscitation. Resuscitation 2015:96:328-40.

9 Australian Bureau of Statistics. Victoria (S/T), 2012. http://stat.abs.gov.au/itt/r.jsp? RegionSummary\&region=2\&dataset=ABS_NRP9_ASGS\&geoconcept=REGION\& measure=MEASURE\&datasetASGS=ABS_NRP9_ASGS\&datasetLGA=ABS_NRP9_ LGA\&regionLGA=REGION\&regionASGS=REGION (cited 5 May 2015).

10 Beck B, Bray JE, Cameron P, et al. Resuscitation attempts and duration in traumatic out-of-hospital cardiac arrest. Resuscitation 2017;111:14-21.

11 Nehme Z, Bernard S, Cameron P, et al. Using a cardiac arrest registry to measure the quality of emergency medical service care: decade of findings from the Victorian ambulance cardiac arrest registry. Circ Cardiovasc Qual Outcomes 2015;8:56-66.

12 Australian Bureau of Statistics. Australian statistical geography Standard (ASGS): Volume 4 -Significant Urban areas, Urban Centres and Localities, section of state: Australian Bureau of Statistics, 2012. Report No: 1270.0.55.004. http://w ww.ausstats.abs.gov.au/Ausstats/subscriber.nsf/0/1080B7CB374FC771CA257 A980013D404/\$File/1270055004_july\%202011.pdf.

13 Deasy C, Bray J, Smith K, et al. Traumatic out-of-hospital cardiac arrests in Melbourne, Australia. Resuscitation 2012;83:465-70.

14 Kuhne CA, Ruchholtz S, Kaiser GM, et al. Mortality in severely injured elderly trauma patients--when does age become a risk factor? World J Surg 2005;29:1476-82.
15 Perdue PW, Watts DD, Kaufmann CR, et al. Differences in mortality between elderly and younger adult trauma patients: geriatric status increases risk of delayed death. J Trauma 1998:45:805-10.

16 Sampalis JS, Nathanson R, Vaillancourt J, et al. Assessment of mortality in older trauma patients sustaining injuries from falls or motor vehicle collisions treated in regional level I trauma centers. Ann Surg 2009;249:488-95.

17 Kleber C, Giesecke MT, Lindner T, et al. Requirement for a structured algorithm in cardiac arrest following major trauma: epidemiology, management errors, and preventability of traumatic deaths in Berlin. Resuscitation 2014;85:405-10.

18 Iwami T, Nichol G, Hiraide A, et al. Continuous improvements in "chain of survival" increased survival after out-of-hospital cardiac arrests: a large-scale population-based study. Circulation 2009;119:728-34.

19 Smith JE, Rickard A, Wise D. Traumatic cardiac arrest. J R Soc Med 2015;108:11-16.

20 Barnard E, Yates D, Edwards A, et al. Epidemiology and aetiology of traumatic cardiac arrest in England and Wales - A retrospective database analysis. Resuscitation 2017;110:90-4.

21 McDermott FT, Cooper GJ, Hogan PL, et al. Evaluation of the prehospital management of road traffic fatalities in Victoria, Australia. Prehosp Disaster Med 2005;20:219-27.

22 Idris AH, Berg RA, Bierens J, et al. Recommended guidelines for uniform reporting of data from drowning: the "Utstein style". Circulation 2003;108:2565-74.

23 Ringdal KG, Coats TJ, Lefering $R$, et al. The Utstein template for uniform reporting of data following major trauma: a joint revision by SCANTEM, TARN, DGU-TR and RITG. Scand J Trauma Resusc Emerg Med 2008;16:7.

24 Australian Resuscitation Council. ANZCOR Guideline 11.10.1 - Management of cardiac arrest due to trauma. https://resus.org.au/guidelines/ (accessed 6 Jan 2016).

25 Truhlář A, Deakin CD, Soar J, et al. European Resuscitation Council guidelines for resuscitation 2015: section 4. cardiac arrest in special circumstances. Resuscitation 2015;95:148-201. 